\title{
Performance analysis of interactive multimodal CME retraining on attitude toward and application of OPCAB
}

\author{
Alexander Albert, MD, ${ }^{a}$ Eric A. Peck, MD, ${ }^{\mathrm{b}}$ Patrick Wouters, MD, PhD, ${ }^{\mathrm{c}}$ Jan Van Hemelrijck, MD, PhD, ${ }^{\mathrm{c}}$ \\ Christophe Bert, $\mathrm{MD}, \mathrm{PhD}^{\mathrm{c}}$ and Paul Sergeant, $\mathrm{MD}, \mathrm{PhD}^{\mathrm{a}}$
}

See related editorial on page 14.

From the Department of Cardiac Surgery, University Hospital Gasthuisberg, Leuven, Belgium, ${ }^{a}$ the Division of Cardiac Surgery, The Johns Hopkins Hospital, Baltimore, $\mathrm{Md}^{\mathrm{b}}{ }^{\mathrm{b}}$ and the Department of Anesthesia, University Hospital Gasthuisberg, Leuven, Belgium.

The Katholieke Universiteit of Leuven, Belgium, had an educational agreement with Medtronic and Guidant, covering the studied CME activity and for most of the visiting teams. Both industrial partners had no interaction with study design, data collection, data analysis, or manuscript

Received for publication June 24, 2005; accepted for publication Aug 29, 2005.

Address for reprints: Prof Dr Paul Sergeant, Cardiac Surgery Department, Gasthuisberg University Hospital, Herestreet, B 3000, Leuven, Belgium (E-mail: paul.sergeant@uz. kuleuven.ac.be)

J Thorac Cardiovasc Surg 2006;131:154-62 $0022-5223 / \$ 32.00$

Copyright () 2006 by The American Association for Thoracic Surgery

doi:10.1016/j.jtcvs.2005.10.004
Objective: The transfer of tacit and codified knowledge on a surgical technique is studied in a consecutive cohort of teams participating in interactive multimodal continuing medical education (CME) retraining in off-pump coronary artery bypass (OPCAB).

Methods: Fifty teams of $1.3 \pm 0.5$ surgeons and $1.1 \pm 1.9$ anesthetists visited 2.2 \pm 0.7 days. Variables describe the pre-visit cardiac activity and OPCAB attitude, complexity score (10 frequently cited complexity criteria), application, and conversion rate. The multimodal approach to knowledge transfer included interactive discussions (commitment; resistances; levers and process of change; methods; outcome; resource optimization), active participation in $3.8 \pm 1.3$ unselected cases (anchor-stitch, enucleation techniques), low-fidelity bench model (shunt placement, anastomotic technique), and CD-ROM. Exit end points included OPCAB attitude and complexity score. Late end points (3 months) included OPCAB attitude, complexity score, and application rate.

Results: OPCAB was considered, upon exit, beneficial for all patients by $90 \%$ of the teams (versus $29 \%$ pre-visit), but by only $62 \%$ of the teams at 3 months. The complexity score downgraded at exit from $3.6 \pm 2$ (pre-visit) to $1.2 \pm 1(P<.001)$ but increased again at 3 months to $1.6 \pm 1(P=.001$ versus pre-visit and $P=.001$ versus exit). The 3 -month OPCAB rate of the surgeons was $49 \% \pm 32 \%$ versus $23 \%$ $\pm 28 \%$ pre-visit $(P<.0001)$. This was influenced by the pre-visit OPCAB rate and education, as well as by the post-visit changes in complexity scores and attitude. The conversion rate toward cardiopulmonary bypass improved from $3.5 \% \pm 5 \%$ (previsit) to $1.3 \% \pm 3 \%$ (3 months, $P=.006$ )

Conclusions: The multimodal OPCAB re-training resulted in a substantial increase of the application, concomitant with a decrease in conversion. The positive impact on attitude and complexity score, at exit, was somewhat reduced in the following clinical confrontation.

$\mathrm{T}$ he first coronary bypass operation $(\mathrm{CABG})$ was performed without the assistance of extracorporeal circulation ${ }^{1}$ (ECC). The technique was exceedingly challenging with an unstable surgical field and the constant threat of ischemic complications and the risk of a technically inferior anastomosis. With the advent of the cardiopulmonary bypass circuit, CABG procedures were safer, repeatable, and teachable. In recent years, industry has developed devices allowing a safe, high-quality CABG procedure to be performed without the assistance of ECC. Many surgeons have adopted this technique of off-pump coronary bypass (OPCAB) as part of their surgical armamentarium or as a new concept applicable to all CABG patients. Some units ${ }^{2}$ have taken a years-long and gradual approach to implementing this new technology by gradually expanding the patient-spectrum and surgeons; other teams have used retraining and guidance by expert centers to accelerate this 

Abbreviations and Acronyms
$\mathrm{CABG}=$ coronary artery bypass grafting
CME = continuing medical education
$\mathrm{CMEST}=$ Continuing Medical Education of Surgical Technologies
$\mathrm{ECC}=$ extracorporeal circulation
OPCAB = off-pump coronary artery bypass
SWOT $=$ strengths, weaknesses, opportunities, threats

process. Very few cardiac surgeons have been trained in these techniques during their residency program. ${ }^{3}$ The unique technical challenges of OPCAB grafting have raised concern that adoption of OPCAB may lead to poorer outcomes during each surgeon's learning curve. ${ }^{4,5}$ As yet, studies have focused on training residents ${ }^{6-8}$ in the OPCAB procedure whereas limited study has aimed on how to most effectively retrain experienced cardiac surgeons.

The Katholieke Universiteit of Leuven reengineered toward OPCAB in all patients and for all surgeons in October 1999. 9,10 Since that date, it provided OPCAB training and retraining, as well, for residents as for experienced surgeons in different formats, based on well-studied and defined educational backgrounds. Between January 2000 and January 2004, 294 multimodal individualized trainings, given to 379 different surgeons and anesthetists, preceded this study. It is the intention of this study to investigate the performance of this format and provide a model and guidance for similar educational venues on the application of this or other new technologies.

\section{Materials and Methods \\ Study Design}

Fifty consecutive visiting teams were enrolled in the study from February through November 2004. Informed consent was obtained from the contact individual for each group. Data were collected by means of three questionnaires.

The initial data form (Appendix 1) was completed on the team's arrival at the hospital, prior to any interaction. This included their OPCAB benefit attitude and their OPCAB complexity score. The OPCAB benefit attitude identified the spectrum of patients (low-risk, high-risk, all patients) they believed receive the most benefit from OPCAB. The OPCAB complexity score identifies 10 anatomic and physiologic conditions commonly considered complexity variables for OPCAB. These included redo operation, the presence of left main disease, severe left ventricular hypertrophy, low ejection fraction, intramural or intraseptal coronary vessels, posterolateral wall coronary vessels, sequential arterial grafts, recent infarct (within 7 days), and an unstable ST segment. The total number of positive responses was calculated as "complexity score." After the visit, the Exit Questionnaire (see Appendix 2) was completed. The 3-Month Follow-Up Questionnaire (Appendix 3) gave insight into the late performance after the training.

\section{Educational Basis}

The Leuven CMEST (Continuing Medical Education of Surgical Technologies) has been based, over the years preceding the current analytic project, on the science of knowledge management and technology implementation. Three core elements formed its basis: the taxonomy of learning objectives, the theory of adult learning, and the dynamic theory of organizational knowledge creation.

The taxonomy of learning objectives ${ }^{11}$ identifies knowledge, skills, and attitude. Knowledge is gained by the digestion of information and its transformation through the process of learning. Skills require the development of psychomotor competencies, a process based on regular practice, expertise motivation, and ongoing training. Attitude refers to how knowledge and skills are combined in the care of patients, including clinical judgment, decision making, and the values of professional behavior.

The theory of adult learning ${ }^{12}$ integrates cognitive and individualized elements. Cognitive elements ${ }^{13}$ focus on internal mental processes, solving real problems. Therefore, perception, reasoning, and understanding are valued more than memory. CMEST was individualized ${ }^{14}$ because of the different stages of experience preceding the training, the different expectations, and the individual learning styles.

The dynamic theory of organizational knowledge $e^{15}$ explains the process from knowledge creation by the individual to its integration within the organization. Codified knowledge ${ }^{16}$ refers to knowledge that is transmittable in formal, symbolic language, whereas tacit knowledge is hard to articulate, acquired through experience or participation, often context specific. The interaction between surgery and anesthesia is a typical example of tacit knowledge. Tacit and codified knowledge exist along a spectrum and are not mutually exclusive. The transfer of these different natures of knowledge can be through socialization (tacit-tacit), through combination (codified-codified), through externalization using metaphors and analogies (tacit-codified), or through traditional learning methods (codified-tacit).

\section{Knowledge Transfer Process}

The knowledge transfer process, consistent over the study interval, was structured in a mind map. The faculty consisted of one surgeon and, alternating, one of three senior anesthetists. After completion of the entry form, well-defined learning objectives were discussed in the first hours of the first day and before surgery. The learning objectives started with a SWOT analysis (strengths, weaknesses, opportunities, threats) of CABG, the need for, the process, and the management of change. This was followed by a discussion of the reengineering: multidisciplinary interaction, monitoring, anesthetic management and conditioning, surgical technique subdivided in teachable components, and finally the actual implementation for all patients and all surgeons. This discussion was guided by slide presentations, animations, and video sequences. Subsequently, the first day, the surgeons scrubbed in and the anesthetists participated in two unselected OPCAB cases, performing well-defined teaching objectives: multidisciplinary interaction, anesthetic management, deep stitch placement, cardiac enucleation, and manipulation. The sequential approach of the retraining is best demonstrated by the enucleation technique. It is first described verbally, then in animation, followed by video, by expert cognitive modeling on the human in the operating theater, 
TABLE 1. Team and retraining variability

\begin{tabular}{lc}
\hline \multicolumn{1}{c}{ Number of teams (N) } & $\mathbf{5 0}$ \\
\hline Visiting team structure & $1.3 \pm 0.5$ \\
Surgeons & $1.1 \pm 1.9$ \\
Anesthetists & $0.1 \pm 0.2$ \\
Nurses & \\
Originating unit & 647 cases (62\% of cardiac \\
CABG annual N cases (\% of & activity) \\
total cardiac activity) & $19 \%$ (range $0-100 ;$ median $14 \%$ ) \\
& $3.4 \% \pm 5 \%$ (range $0-20$ ) \\
OPCAB activity (\% of CABG) & \\
OPCAB conversion rate & \\
toward CPB (\% of & \\
OPCAB) & \\
Visiting surgeon & \\
CABG annual N cases & \\
OPCAB activity ( $\%$ of CABG) & $23 \%$ (mean $44 \pm 18$ cases; \\
& median 5 cases) \\
At entry & \\
OPCAB attitude & $29 \%$ beneficial for all \\
& $4 \%$ beneficial for low risk \\
OPCAB complexity score & $67 \%$ beneficial for high risk \\
Retraining activity & $3.6 \pm 2$ \\
Number of days & \\
Number of OPCAB cases & $2.2 \pm 0.7$ \\
Number of conversions & $3.8 \pm 1.3$ \\
seen & $0 \pm 0$ \\
\hline
\end{tabular}

and finally repeated (over the different patients) nonexpert modeling by the visiting surgeon corrected by the expert. The different OPCAB procedures were linked by interactive discussions between expert and scholars about procedural variability and organizational aspects of perfusion and nursing. Virtual training was simultaneously addressed by having team members practice shunt insertion and anastomotic techniques in low-fidelity training models.

Day 2 starts again with expert-scholar interaction about the cognitive and motor skills issues of day 1 , as well as economic aspects, real-time quality control monitoring systems, outcome analysis, and finally early and late outcome data of our own population. The acquired OPCAB knowledge was reinforced with two additional unselected cases. The CME ended with a wrap-up discussing the organizational and psychologic issues the team would encounter at return in their units. The exit report was completed and a CD-ROM with the complete slide presentation and $\mathrm{OPCAB}$ bibliography provided to assist in institutional education.

\section{Team Variability}

Fifty teams of surgeons, anesthetists, and nurses from 16 countries were retrained during 2 days (Table 1). The teams came at their own initiative through word of mouth. The originating institutions were mostly academic $(68 \%)$. The originating units had an average cardiac surgery practice of 1000 cases (range: 175-3375), with 7.0 \pm 4.6 staff surgeons and $1.9 \pm 2.6$ licensed surgeons without staff position. Information about their early post-CABG morbidity and mortality (Appendix 1) seemed unavailable or incomplete in $76 \%$ of the teams, even after repeated requests post hoc. The visiting teams worked together several days a week and some had participated in different formats of OPCAB retraining, preceding the current one. Complex enucleation methods, using sling techniques in combination with apical suction and anastomotic area stabilization, were less commonly used (34\%); routine shunting was more commonly used (60\%). The average OPCAB application rate of the staff surgeons not participating in the training was only $8 \%$ before training, whereas it was $23 \%$ for the visiting surgeons. The most frequently cited teaching expectation was multivessel arterial OPCAB revascularization $(90 \%)$.

Most teams were uncertain about the beneficial effect of OPCAB, as well, on target population as on specific outcome improvement. There was no correlation between pre-CMEST OPCAB attitude and OPCAB application.

The OPCAB complexity score (Table1 and Figure 1) cited most frequently intraseptal and intramural vessels, and unstable ST segment as OPCAB complexity variables.

The team variability influenced the Complexity Score at entrance $\left(\mathrm{R}^{2}=.37 ; P<.001\right)$ : Surgeons not using a sling support combined with an apical suction device had more contraindications for OPCAB, as well as those working in smaller units (less than 1000 cases/year). In contrast, surgeons with a higher annual rate of CABG and surgeons with a history of OPCAB education (interactive discussion, live demonstration) had fewer contraindications.

The decision about retraining was taken by the individual in $64 \%$, by the team in $22 \%$, and by the departmental director in $14 \%$ of the teams. Only $4 \%$ of the teams funded the retraining expenses (travel and lodging) from personal or institutional resources; the other teams were supported by the industry. The industry signed an educational agreement with the K. U. Leuven.

\section{Follow-up and Statistical Analysis}

Late follow-up was complete for all teams, but labor-intensive (average $>5$ contacts). Additional variability was calculated indirectly from available variability (eg, the average OPCAB application rate in a unit of the nonparticipating staff surgeons). The OPCAB attitude analysis used matched-pair analysis, after ordinal transformation. The analysis applied univariate and multivariate non-time-related methods (linear and logistic regression) and variable transformations (eg, nominal into ordinal, continuous into nominal, and continuous into mathematical transformations) to obtain optimal relations between studied outcomes and variability.

\section{Results \\ At Exit}

The OPCAB attitude at exit was $(P<.001)$ influenced by the training; OPCAB was considered beneficial for all patients by $90 \%$, for the low-risk patients only by $2 \%$, and for the high-risk patients only by $6 \%$ of the teams, and $2 \%$ were uncertain. No teams reduced (pretraining versus at exit) the beneficial group level from all to a smaller subset. The OPCAB complexity score at exit was $1.24 \pm 1$, strongly reduced $(P<.001$, mean difference -2.4$)$ versus at entry. 


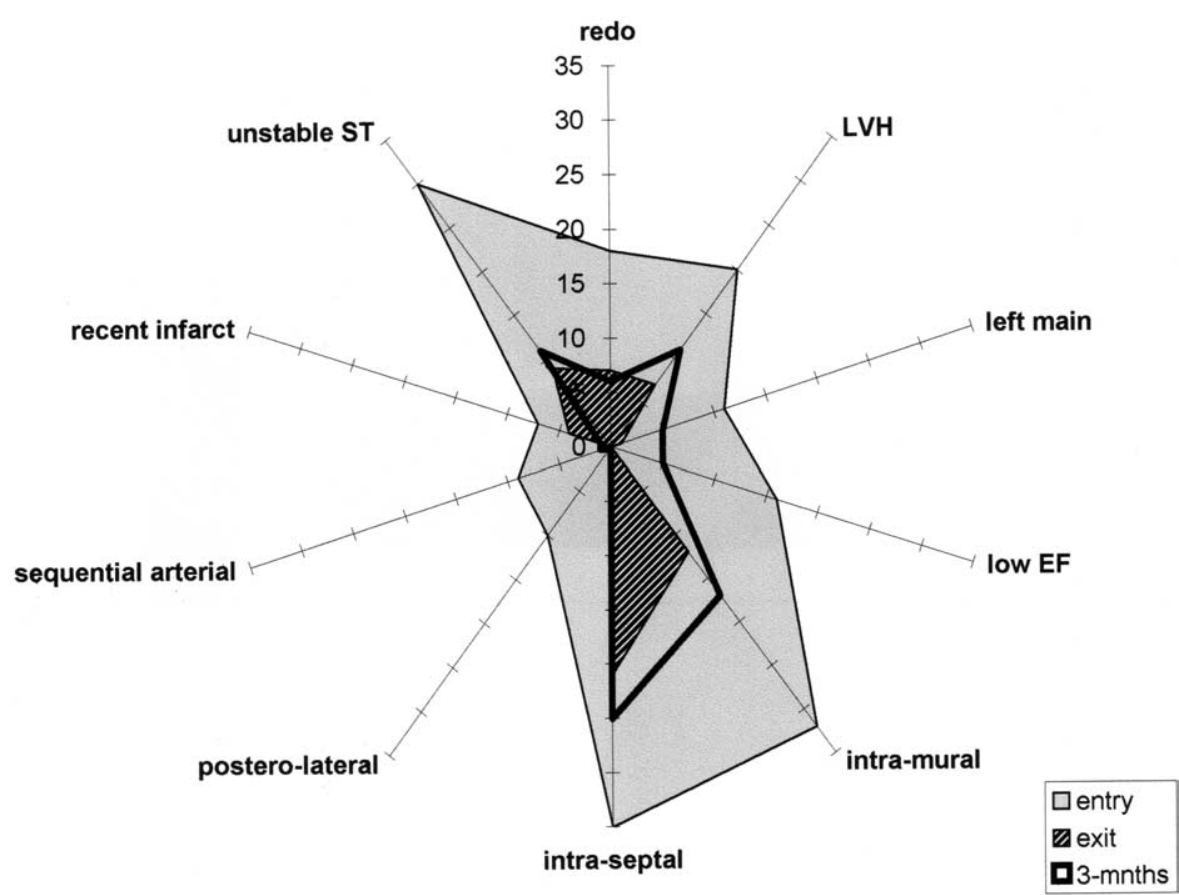

Figure 1. The 10 most frequently cited complexity criteria, by their frequency, cited at entry, at exit. and at 3 months. $L V H$, Left ventricular hypertrophy; $E F$, ejection fraction.

The OPCAB complexity variables most frequently cited at exit were intraseptal vessels and intramural vessels. Figure 1 depicts these 10 most frequently cited complexity criteria versus their prevalence before the retraining. The inferolateral and posterolateral location of coronary anastomoses (10 teams at entry), as well as the sequential arterial grafting ( 9 teams at entry) and low ejection fraction (16 teams at entry), disappeared from the list at exit.

\section{At 3 Months}

The OPCAB attitude at 3 months remained strongly influenced by the training ( $P<.001$, versus at entry). OPCAB was considered beneficial for "all patients" by $64 \%$, for the low-risk patients by $10 \%$, and for the high-risk patients by $26 \%$ of the teams. Fifteen teams reduced the beneficial group from "all" to a smaller subset during follow-up (6 teams versus pretraining). The OPCAB attitude at 3 months ("benefit for all") could be predicted reliably (receiver operating characteristic $=0.92$ ) by variables describing the team and the CMEST variability: the size of the clinic ("number of cases"), the pretraining participation in interactive discussions about OPCAB, the CMEST duration (more than 1 day), the CMEST team membership of an anesthesiologist, and the extent of complexity score reduction (at exit versus pre-CMEST).

The complexity score increased slightly at 3 months, versus the exit information, to $1.6 \pm 1(P<.001)$ but was still significantly lower than the complexity score before OPCAB retraining (mean difference: $-2.0 ; P<.001$ ). The retraining variability was related to the decrease in complexity score at 3 months: the number of clinical cases participated in during the retraining and the deletion at exit of intraseptal and intramural vessels as OPCAB complexity criteria $\left(\mathrm{R}^{2}=0.66\right)$. The OPCAB complexity variables most frequently cited at 3 months were: intraseptal vessels and intramural vessels. Figure 2 depicts the 10 most frequently cited complexity criteria versus their prevalence before the retraining and at exit. The posterolateral location of coronary anastomoses remained absent from the list.

The OPCAB application rate of the surgeons increased about $26 \%$ (absolute increase) or $110 \%$ (relative increase) 3 months after the OPCAB retraining $(49 \% \pm 32 \%$ versus $23 \% \pm 28 \%$ pre-visit; $P<.0001)$. The average OPCAB application rate of the staff surgeons who did not participate in the training did not change during the 3-month interval $(8.2 \%$ versus $10.3 \% ; P=.21)$.

The increase of the OPCAB application rate after training could be estimated by pre-visit variability; thus, surgeons mainly doing CABG and those who had already attended large audience OPCAB education programs increased their OPCAB rates, whereas surgeons who had been already performing more than $80 \%$ of their CABGs off- 


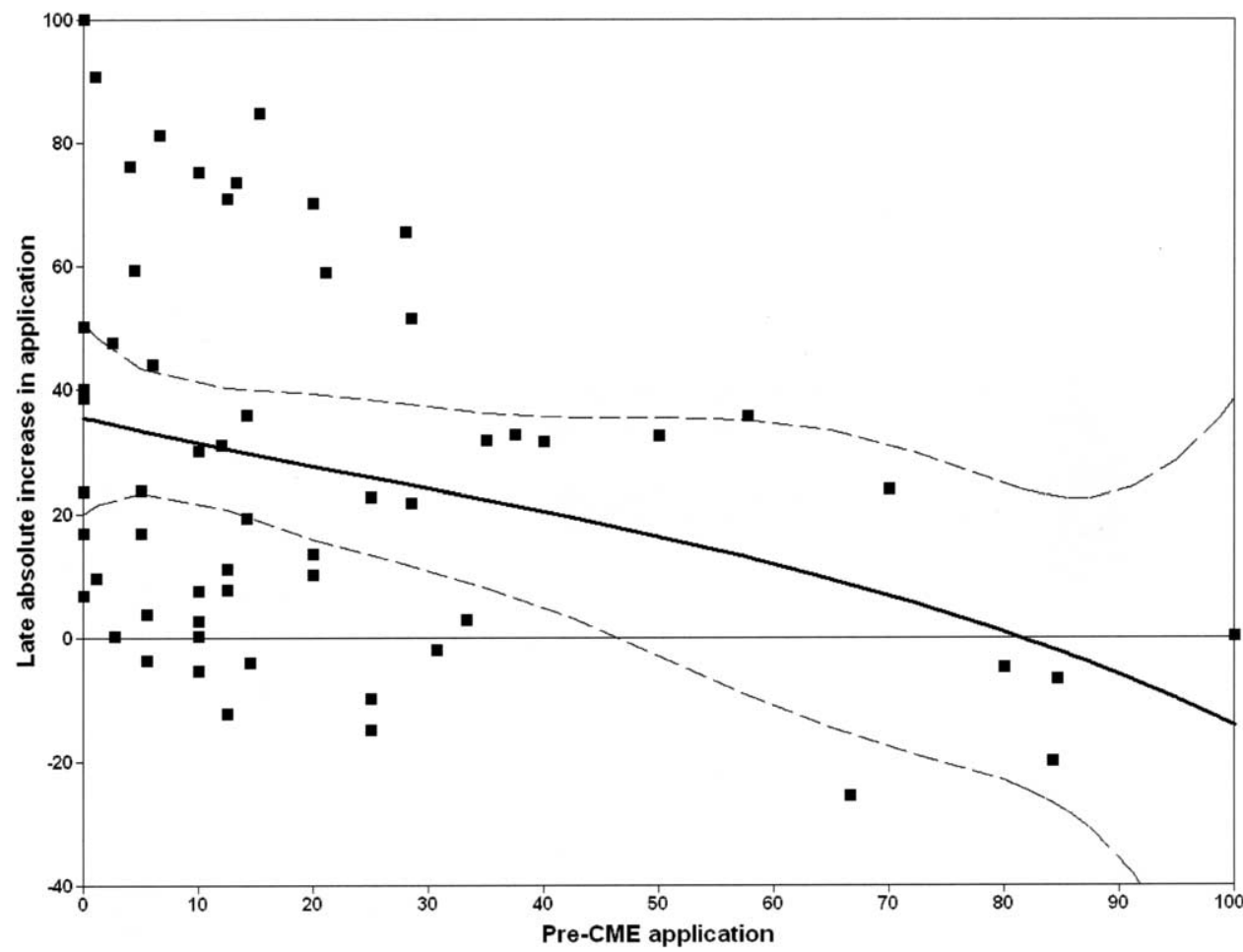

Figure 2. The 3-month increase or decrease in OPCAB application rate, the polynomial transformation and its uncertainty, is plotted versus the pretraining application rate. CME, Continuing medical education.

pump did not further increase their OPCAB rates after the training. The pretraining attendance of video-transmitted live demonstrations (unrelated to the educational process studied in this manuscript) had a negative impact on the 3 -month OPCAB increase. The predictive model was further improved by a CMEST-associated variable: the deletion of intraseptal coronary location as an OPCAB complexity (Table 2). It turned out that some surgeons with no or very limited OPCAB experience were able to perform nearly all cases off-pump in the weeks after our CMEST. The 3-month increase and decrease of the OPCAB application rate is plotted versus the pretraining application rate (Figure 2). A significant correlation was found between OPCAB attitude at 3 months and OPCAB application $(P<.001)$.
The conversion rate to $\mathrm{CPB}$ improved from $3.5 \% \pm 5 \%$ (pre-visit) to $1.3 \% \pm 3 \%(P=.006)$.

\section{Discussion}

Limitations of the Dataset and of the Analysis

The analysis is limited by the size and the variability in the dataset. The team as well as the individualized approach was based on earlier CMEST experience in Leuven (see Methods section), as was the 2-day optimal duration. The analysis was no randomized controlled trial because no visiting team would agree to be the placebo team.

Before one can study the effect of additional variability, one has to build evidence about a basic effect of CMEST. This was missing about OPCAB application and conversion. We have tried to create indirect information about the

TABLE 2. Pretraining and training-associated factors influencing the increase of the OPCAB rate of the surgeons during a 3-month interval after training (multivariate linear regression analysis, $P<.001, R^{2}=0.46$ )

\begin{tabular}{lrr}
\hline Variable & Estimate & P value \\
\hline + Effect of pre CMEST CABG focus of surgeon (in \% of total activity) & 76.3 & .0062 \\
+ Effect of participation pre CMEST in OPCAB education (large audience) & 12.9 & .0018 \\
- Effect of pre CMEST OPCAB rate >80\% & -22.3 & .0057 \\
- Effect of attending pre-CMEST an OPCAB live demonstration & -12.9 & .018 \\
- Effect of citing "intraseptal coronary" at exit as a complexity criterion & -6.4 \\
\hline
\end{tabular}


nonvisiting members of a team and have used them as a control group. The analysis includes only 50 teams (70 surgeons), thereby limiting the multivariate correcting capacity.

The reported late application rates were not validated by an independent observer, but there is strong evidence ${ }^{17}$ that self-reports of behavior change accurately represent the actual change after CME courses.

\section{Attitude Toward OPCAB}

Attitude, a cognitive variable, has been documented ${ }^{18}$ to be one of the most important drivers toward change. Selfmotivation serves as a driving force to absorb knowledge. Gardner ${ }^{19}$ describes 7 important elements influencing the process of change of the individual: reason, research, resonance, representational redescriptions, resources/rewards, real world events, and resistances. Reason or attitude implies that an individual is able to see a logical, rational approach to the pertinent issue and weigh each consideration appropriately. A surgeon would be able, for example, to evaluate each risk and benefit of the OPCAB procedure as it compares with conventional CABG surgery and make an honest assessment as to whether to change his or her belief.

The attitude toward OPCAB in the retrained surgeons was strongly improved by the training as well at exit as at the late evaluation, although somewhat less. The OPCAB attitude at 3 months ("benefit for all") could be predicted reliably, aside through other factors, by training elements (duration of the training more than 1 day, the presence of an anesthesiologist in the team, and extent of complexity score reduction during training).

\section{Complexity Scoring}

Complexity scoring is based on cognitive and motor skills, since previous OPCAB experience and exposure will influence this scoring. Surgeons not combining a sling support with an apical suction device and those without OPCAB education experience had more complexity variables at entry.

The scholars were exposed during their training interval to different surgical complexities, addressed as well by the expert as by themselves under the corrective supervision of the expert. Custers and associates ${ }^{20}$ and Banderas ${ }^{21}$ identified that the effect of this exposure is maintained and even accumulated across repeated exposures, providing observers with opportunities to discern the structure of the modeled actions, to organize and verify what they know, and to give special attention to problematic aspects in subsequent exposures.

The score-reducing effect, at exit, of the training remained stable at 3 months. The posterolateral location of coronary anastomoses disappeared permanently from the list of complexity criteria. The most frequently cited variables at 3 months, intraseptal and intramural coronary anatomy, are already challenging for regular CABG. The prevalence of certain of these complexity criteria in the limited number of attended procedures during the training certainly affected this complexity score. However, surgeons who were not considering an intraseptal coronary as complexity variable for $\mathrm{OPCAB}$ at end of the training were more likely to increase their OPCAB rates.

\section{Application and Conversion}

The final purpose of a retraining is an improved benefit for the patient. This needs to be preceded by a widened application in the spectrum of patients carrying improvable risk. This study has identified a highly significant increase over a short interval. Nonparticipating surgeons from the same unit did not increase at all (control group), indicative of disappointing absence of knowledge transfer to the colleagues within the unit. Educational material provided was seldom shared within the unit, according to the open questionnaires. The predictive model of the 3-month application rate included characteristic elements of OPCAB retraining (attitude, complexity score). A great variability in training effect was identified. Some surgeons with minimal OPCAB experience before training performed $\mathrm{OPCAB}$ in the majority of their patients, a target not reached by $50 \%$ of the visiting surgeons.

The appearance of cognitive variables such as attitude and complexity scores in the multivariate model is additional proof of the major importance of cognitive elements in the process of change.

The K.U.Leuven OPCAB retraining model focuses on a team approach. Literature about technology implementation has strongly recommended such an approach when tacit knowledge was deeply imbedded in an interpersonal interaction. This is certainly valid for OPCAB application, where this can improve coordination ${ }^{22}$ and reduce complications during the initial experience, ${ }^{23}$ and our analysis confirms observations made in unrelated domains. Virtual training models are evaluated in fidelity (similar) to reality (the human heart). Low-fidelity training models are known to be as beneficial ${ }^{24}$ to surgical learning as high-fidelity models. Low-fidelity virtual training models are enthusiastically used in the K.U.Leuven resident training and were actively demonstrated to the visiting scholars; only 6 teams actually built such a simple model and practiced with them. This confirms the literature ${ }^{25}$ identifying the reluctance of model practicing in surgical trainees. The pretraining experience of a live procedure seemed to have had a lasting negative effect on the posttraining application rate. It is certain that most live procedures fail on aspects of education and technology transfer. The lasting negative effect is dis- 


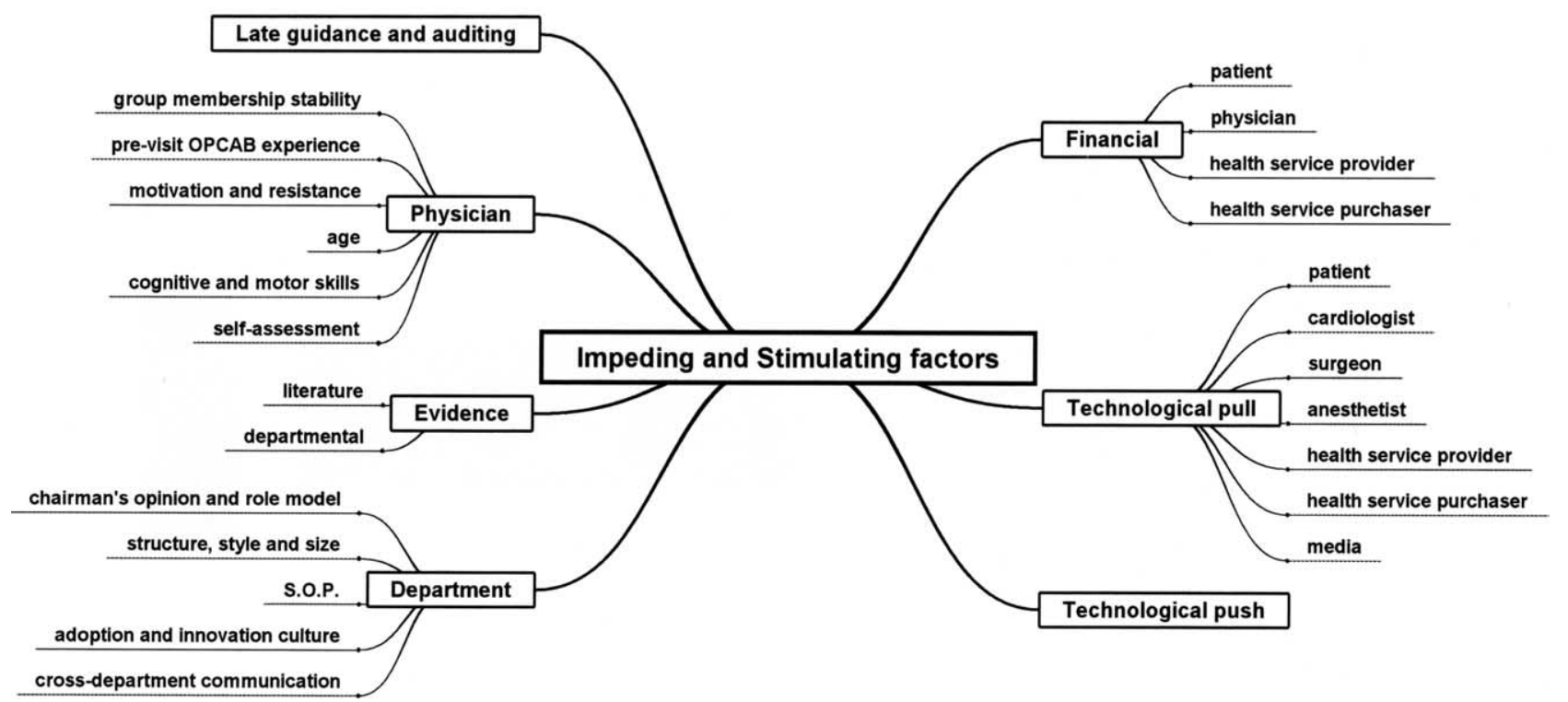

Figure 3. The mind map of the impeding and stimulating factors after retraining. OPCAB, Off-pump coronary artery bypass; S.O.P., standard operating procedure.

turbing and probably needs reconfirmation in additional trials.

Figure 3 structures the most frequently cited impeding and stimulating factors in the application of a new technology, as identified in scientific literature. The size and the specialization of the originating institution determined the complexity score, the OPCAB attitude, and/or the OPCAB application rate. This has been similarly identified in laparoscopic techniques where size of the clinic and the specialization stimulated the adoption. ${ }^{26,27}$ The seniority of the visiting teams had no effect on the posttraining application. No correlation of the years since surgical license and the OPCAB application was observed in a study about adoption of OPCAB in Canada. ${ }^{28}$ More experienced surgeons should have a greater capacity for knowledge acquisition and integration, but their return to investment decreases with the number of years left in practice. ${ }^{27}$ While Desai and colleagues $^{28}$ observed a significant correlation between OPCAB attitude and OPCAB application, we found this correlation only after the training rather than before the training. Furthermore, we still recognize in many surgeons the apparent discrepancy between a positive OPCAB attitude and a low application rate. The OPCAB attitude is not the only influencing factor. In addition to the previous mentioned factors, there is also an important role of the departmental chairman, according to our questionnaire, either as a formal opponent (veto in 6 teams) or sometimes as an absence of role modeling. There was no pretraining written commitment of the chairman to implement the technology in his unit after the training, but this might have been a limitation of this CME. The veto of the chairman was the impeding factor in $55 \%$ of the nonstarters after a laparoscopic $^{29}$ course. The chairman has to provide the psychologic safety ${ }^{30}$ in the application of new technologies.

\section{Training Team Stability and Expertise}

A possible bias of the late results of this study is the team stability and expertise. The surgical expertise was very variable, often not exceeding the $100 \mathrm{CABG}$ cases a year. This was indicative of smaller cardiac surgery programs, a large number of consultants, or absence of superspecialization in coronary surgery. The superspecialization in cardiac anesthesia was absent in many of our anesthesia scholars, who frequently rotated as a consultant in the different domains of anesthesia. One can expect that these anesthetists would be less familiar with the hemodynamic issues of OPCAB anesthesia. This was insufficiently documented in the pretraining variability. In addition, for departmental reasons, some visiting teams were never allowed to interact in clinical OPCAB cases after their return. A final aspect of the team impact could have been that the entry and exit questionnaires were completed in the presence of the complete team, but this might not have been valid for the 3 -month questionnaire.

\section{Conversion Rates}

The reduction of the conversion rate concomitant with the increase in application after this multimodal retraining is an identifiable improvement in quality of care. A conversion toward ECC, sometimes as frequent as $13 \%$, has been 
associated with increased mortality ${ }^{31}$ and morbidity. Edgerton and coworkers ${ }^{32}$ have identified limited experience in $\mathrm{OPCAB}$ technique as an important predictor towards conversion.

\section{Conclusion}

CMEST is a very intricate process involving an in-depth understanding of the nature of knowledge and the mechanisms of its transfer. An individualized cognitive approach, based on labor-intensive and interactive discussions, team participation, repeated observations, and hands-on practices, can affect the application of a new technology. The impeding effects of institutional variables could be countered by pre-visit discussions about departmental commitments to change. Further analysis is mandatory about improved pre-visit testing and teaching, superior team selection, mandatory written commitment by the chairman, the feasibility and benefit of extending this OPCAB CMEST beyond the 2-day interval, the benefit of a mandatory participation by the chairmen of cardiac surgery and anesthesia, the extended exercise on low-fidelity models, and other possible improvements.

\section{References}

1. Kolessov VI. Mammary artery-coronary artery anastomosis as method of treatment for angina pectoris. J Thorac Cardiovasc Surg. 1967;54:535-44.

2. Song HK, Petersen RJ, Sharoni E, Guyton RA, Puskas JD. Safe evolution towards routine off-pump coronary artery bypass: negotiating the learning curve. Eur J Cardiothorac Surg. 2003;24:947-52.

3. Ricci M, Karamanoukian HL, D'Ancona G, DeLaRosa J, Karamanoukian RL, Choi S, et al. Survey of resident training in beating heart operations. Ann Thorac Surg. 2000;70:479-82.

4. Bonchek LI. Off-pump coronary bypass: Is it for everyone? J Thorac Cardiovasc Surg. 2002;124:431-4.

5. Mack M, Bachand D, Acuff T, Edgerton J, Prince S, Dewey T, et al. Related improved outcomes in coronary artery bypass grafting with beating-heart techniques. J Thorac Cardiovasc Surg. 2002;124:598607.

6. Caputo M, Bryan AJ, Capoun R, Mahesh B, Ciulli F, Hutter J, et al. The evolution of training in off-pump coronary surgery in a single institution. Ann Thorac Surg. 2002;74:S1403-7.

7. Karamanoukian HL, Panos AL, Bergsland J, Salerno TA. Perspectives of a cardiac surgery resident in-training on off-pump coronary bypass operation. Ann Thorac Surg. 2000;69:42-5.

8. Jenkins D, Al Ruzzeh S, Khan S, Bustami M, Modine T, Yacoub M, et al: Multi-vessel off-pump coronary artery bypass grafting can be taught to trainee surgeons. Heart Surg Forum. 2002;5 Suppl 4:S34254.

9. Sergeant P, Wouters P, Meyns B, Bert C, Van Hemelrijck J, Bogaerts $\mathrm{C}$, et al. OPCAB versus early mortality and morbidity: an issue between clinical relevance and statistical significance. Eur J Cardiothorac Surg. 2004;25:779-85.

10. Sergeant P, de Worm E, Meyns B, Wouters P. The challenge of departmental quality control in the reengineering towards off-pump coronary artery bypass grafting. Eur J Cardiothorac Surg. 2001;20: 538-43.

11. Kneebone R. Simulation in surgical training: educational issue and practical implications. Med Educ. 2003;37:267-77.

12. O'Brien DD, Freemantle N, Wolf FM, Mazmanian P, Taylor-Vaisey A. Impact of formal continuing medical education. JAMA. 1999;282: 867-74.
13. Hall JC, Ellis C, Hamdorf J. Surgeons and cognitive processes. $\mathrm{Br} J$ Surg. 2003;90:10-6.

14. Lane NE. Skill acquisition rates and patterns, issues and training implications. New York: Springer Verlag; 1987.

15. Nonaka I. A dynamic theory of organizational knowledge creation. Organization Sci. 1994;5:14-37.

16. Polanyi M. The tacit dimension. Garden City (NY): Doubleday; 1966.

17. Curry L, Purkis I. Validity of self-reports of behaviour changes by participants after a CME course. J Med Educ. 1986;61:579-84.

18. Dirksen CD, Ament AJH, Go P. Diffusion of six surgical endoscopic procedures in the Netherlands: stimulating and restraining factors. Health Pol. 1996;37:91-104

19. Gardner H. Changing minds. Boston: Harvard Business School Press; 2004.

20. Custers EJFM, Regehr G, McCulloch W, Peniston C, Reznick R. The effect of modelling on learning a simple surgical procedure: see one, do one or see many, do one? Adv Health Sci Educ. 1999;4:123-43.

21. Bandura A, Social foundations of thought and actions: a social cognitive theory. Englewoods Cliffs (NJ): Prentice-Hall; 1986.

22. Pisano GP, Bohmer RMJ, Edmondson AC. Organizational differences in rates of learning: evidence from the adoption of minimally invasive cardiac surgery. Manage Sci. 2001;47:752-68.

23. See WA, Cooper CS, Fisher RJ. Predictors of laparscopic complications after formal training in laparascopic surgery. JAMA. 1993;270: 2689-92.

24. Grober ED, Hamstra SJ, Wanzel KR, Reznick RK, Matsumoto ED, Sidhu RS, et al The educational impact of bench model fidelity on the acquisition of technical skill. Ann Surg. 2004;240:374-81.

25. Wanzel KR, Ward M, Reznick RK. Teaching the surgical craft: from selection to certification. Curr Probl Surg. 2002(Jun):583-659

26. Poulsen PB, Vondeling H, Dirksen CD, Adamsen S, Go P, Ament A. Timing of adoption of laparoscopic cholecystectomy in Denmark and in The Netherlands: a comparative study. Health Policy. 2001;55:8595.

27. Escarce JJ, Bloom BS, Hillman AL, Shea JA, Schwartz JS. Diffusion of laparoscopic cholecystectomy among general surgeons in United States. Med Care. 1995;33:256-71.

28. Desai ND, Pelletier MP, Mallidi HR, Christakis GT, Cohen GN, Fremes SE, et al. Why is off-pump coronary surgery uncommon in Canada? Results of a population-based survey of Canadian heart surgeons. Circulation. 2004;47:270-6.

29. Morino M, Festa V, Garone C, Survey on Torino courses. Surg Endosc. 1995;9:46-8.

30. Edmondson AC. Psychological safety and learning behaviour in work teams. Admin Sci Q. 1999;44:350-83.

31. Légaré J-F, Buth KJ, Hirsch GM. Conversion to on pump from OPCAB is associated with increased mortality: results from a randomized controlled trial. Eur J Cardiothorac Surg. 2005;27:296-301.

32. Edgerton JR, Dewey TM, Magee MJ, Herbert MA, Prince SL, Jones $\mathrm{K}$, et al. Conversion in off-pump coronary artery bypass grafting: an analysis of predictors and outcomes. Ann Thorac Surg. 2003;76:113843.

\section{Appendixes}

\section{Appendix 1: Variables of the Entry Form}

Unit: Country; type of hospital (academic, non-academic); number of staff surgeons; number of licensed surgeons; number of surgeons with $\mathrm{OPCAB}$ experience; annual number of isolated $\mathrm{CABG}$, isolated valves, combined $\mathrm{CABG} / \mathrm{valves}$, assist devices, transplants, congenital; number of isolated OPCAB; OPCAB conversion rate; outcome data: stroke rate(overall and high risk), dialysis (overall and high risk), early mortality (hospital and 3 months)

Team: Structure of the visiting team by number of surgeons; anesthetists, and nurses; home interaction of visiting team; experience with modes of OPCAB retraining; expectations; stabiliza- 
tion methods used; shunt use; OPCAB benefit attitude; retraining decision and financing mode; OPCAB complexity score

Each physician: Years licensed; CABG and OPCAB routine

\section{Appendix 2: Variables of the Exit Form}

Team: OPCAB benefit attitude; OPCAB complexity score

Retraining activity: Number of days; number of cases attended, observation of conversion ( $\mathrm{Y} / \mathrm{N})$; team action: deep stitch placement, cardiac manipulation, shunt insertion, low-fidelity training

\section{Appendix 3: Variables of the 3-Month Form}

Unit: Number of isolated CABGs of last 3 months; number of isolated OPCABs of last 3 months; OPCAB conversion rate; late evaluation of their educational material

Team: Structure stability of the visiting team; interdepartmental teaching of surgeon, anesthetist, or nurse; stabilization methods used; shunt use; low-fidelity training experience; OPCAB benefit attitude; OPCAB complexity score; open questions about their OPCAB experience

Each physician: $\mathrm{CABG}$ and $\mathrm{OPCAB}$ routine

\section{Availability of Journal back issues}

As a service to our subscribers, copies of back issues of The Journal of Thoracic and Cardiovascular Surgery for the preceding 5 years are maintained and are available for purchase from Mosby until inventory is depleted. Please write to Mosby, Subscription Customer Service, 6277 Sea Harbor Dr, Orlando, FL 32877, or call $800-654-2452$ or 407-345-4000 for information on availability of particular issues and prices. 